\title{
HRTEM and Nano-Beam Diffraction Analysis of Metal-Molecule Interface
}

\author{
Peng Li*, Jian Chen*, Marek Malac *,, , Haijun Yan*, , Andrew Bonifas*,\#, Richard McCreery*,,\# \\ *National Institute for Nanotechnology, Edmonton, 11421 Saskatchewan Drive, T6G 2M9, Canada. \\ Department of Physics, University of Alberta, Edmonton, T6G 2G7, Canada. Department of Chemistry, \\ University of Alberta, Edmonton, T6G 2R3, Canada \# Dept. of Materials Sci. and Eng., The Ohio State \\ Univ., 2041 College Rd. Columbus, OH, 43210
}

We report results on high resolution (HR) imaging and nano-beam diffraction (NBD) analysis of molecular devices, namely the morphology and structure of interface between the molecular layer and the contact electrode. High resolution TEM (HRTEM) imaging is a direct way to review the morphology of an interface and to measure projected interface roughness. Electron diffraction analysis provides data that allow identifying structure and orientation of crystalline materials. Nano-beam diffraction (NBD) allows local structure and orientation to be determined with a probe size a few nanometers in diameter [1]. Molecular device are composed of a molecular layer attached to metallic electrodes (Figure 1). The characteristic of such molecular device can be dominated by the details of the metal-molecule interface [2]. Therefore characterization of crystal orientation of metal particles, interface roughness and thickness of the molecular layer will help to understand the interaction and diffusion of metal atoms and molecules during processing and its effect on device properties. Combining HRTEM and NBD analysis provides a complete morphological and crystal structure analysis of metal-molecule interfaces. Multilayer (Au-molecule-Si) devices were prepared by grafting Nitroazobenzene (NAB) molecules onto p-doped silicon followed by deposition of a Au film in an electron beam evaporator [3]. Sidewall (Au-molecule) junctions were fabricated through surface diffusion-mediated deposition (SDMD) [4], in which metal atoms were deposited remotely and then allowed diffuse onto the molecular layer. NAB molecules were grafted onto side wall of patterned Pyrolyzed photoresist films (PPF). Au film was then deposited at Au vapor incidence angle between $0^{\circ}-5^{\circ}$ from the sample surface normal, and the overhang of the $\mathrm{Cr} / \mathrm{SiO}_{2}$ etch mask shadowed the molecular layer from direct impingement of both incident gold atoms and radiation from the evaporation source. Electronic contact to the molecular layer occurs through surface diffusion of the deposited Au atoms towards and eventually on top of the molecular layer. Figure 1 shows schematic diagrams of cross section views of Au-NAB-Si and side wall junction samples. TEM samples of both devices were prepared in a Zeiss Nvision SEM/FIB dual beam system. A $30 \mathrm{keV} \mathrm{Ga}$ ion beam was used to cut and mill a TEM lamella with a thickness below $100 \mathrm{~nm}$ suitable for TEM examination. Final polishing was done using $5 \mathrm{keV}$ Ga ion beam to minimize ion beam damage. HRTEM images and NBD (with about $2 \mathrm{~nm}$ beam diameter) were taken on a JEOL 2200 FS TEM equipped with a Schottky gun and in-column omega filter. Figure 2 shows a HRTEM image of $\mathrm{Au} / \mathrm{NAB} / \mathrm{Silicon}$ interfaces. The Au/NAB interface is not flat and the average NAB film thickness is $3.1 \pm 0.6 \mathrm{~nm}$ after Au deposition (compared with as-deposit film thickness of $4.8 \pm 0.2 \mathrm{~nm}$ measured by AFM "scratching" technique), suggesting that there is certain degree of Au penetration into the NAB molecular layer. Alternatively the disagreement between TEM and AFM can be explained by the projection nature of TEM, a surface roughness will result in overlap that is integrated along the beam resulting in apparent reduction of NAB thickness. BFTEM images in Figure 3 show surface diffusion mediated Au on the $\mathrm{PPF} / \mathrm{NAB}$ sidewall, which has different morphology from $\mathrm{Au}$ film deposited on horizontal $\mathrm{SiO}_{2}$ surface. NAB molecular layer is not resolved here due to very weak contrast between PPF and the NAB molecular layer. Crystal orientation of each individual Au particle was characterized by NBD analysis as shown in Figure 4. Preferential orientation of Au was not found for the SDMD-deposited Au. 
References:

[1] M. Malac, M. Beleggia, Y. Taniguchi, R. Egerton, Y. Zhu, Ultramicroscopy 109, p.14 (2009).

[2] H. Haick, O. Niitsoo, J. Ghabboun, D. Cahen, J. Phys. Chem. C 2007, 111, (5), 2318-2329.

[3] H. Yan, The Ohio State University, Ph.D. Thesis, 2009.

[4] A. Bonifas, R. McCreery, submitted.

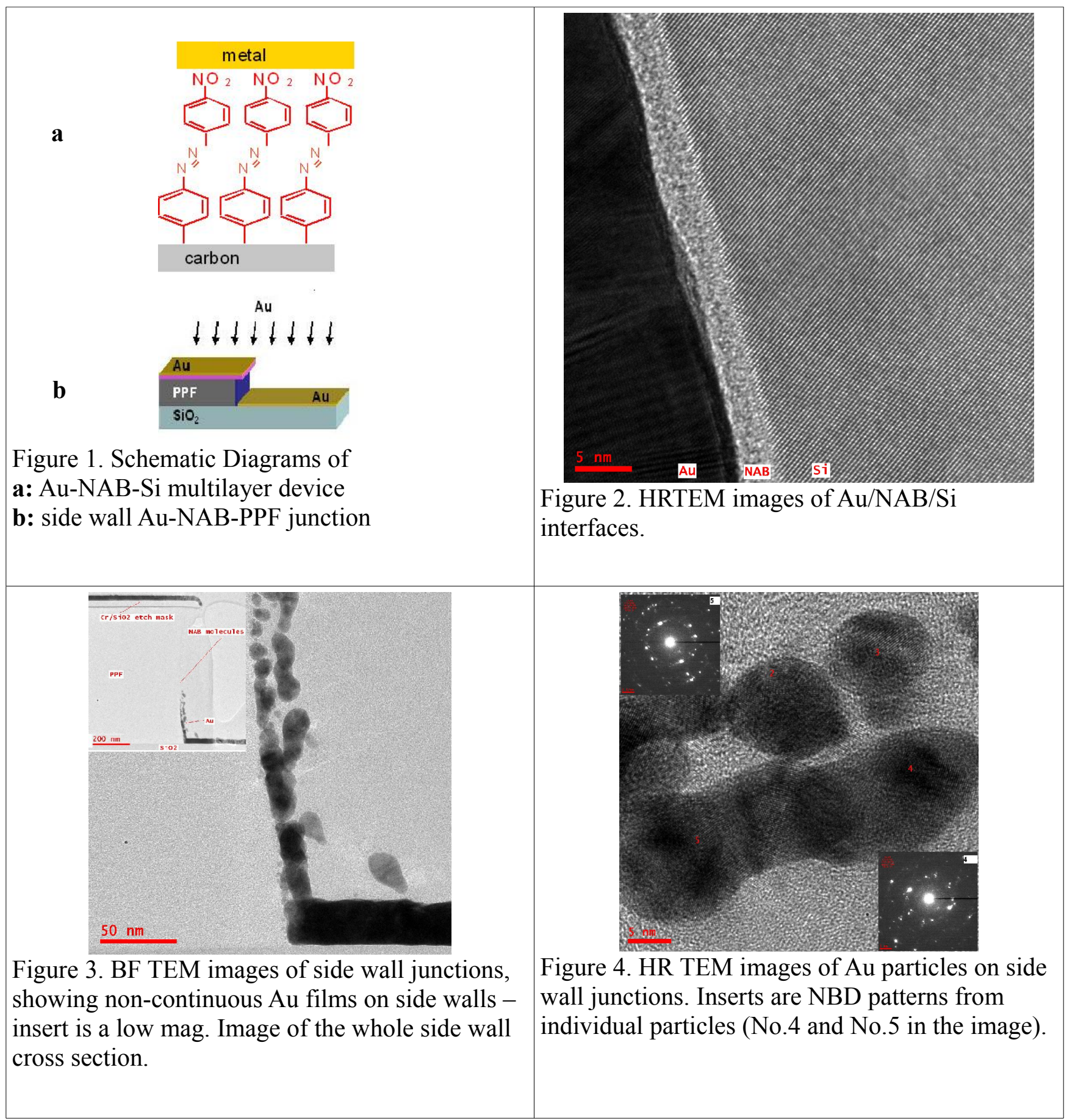

